\title{
OPPORTUNISTIC DIAGNOSIS OF EXTENSIVE PULMONARY EMBOLUS FOLLOWING “COVID-19 BLOOD BATTERY” IN VERY FRAIL OLDER PATIENTS
}

\author{
M.M.C. O’BRIEN' ${ }^{1}$, J.M. MCLOUGHLIN ${ }^{1}$, E.C. MULKERRIN² \\ 1. Galway University Hospital, Galway, Ireland; 2. Galway University Hospital / NUIG, Galway, Ireland. Corresponding author: Dr. Michelle Mary Clare O'Brien, Galway University \\ Hospital, Galway, Ireland, drmichelleobrien@gmail.com, 091-544-000
}

\begin{abstract}
D-dimer is routinely measured to exclude the diagnosis of venous thromboembolism and is its main biomarker. Appropriate age-adjusted D-dimer testing improves D-dimer specificity, could decrease inappropriate CT pulmonary angiograms in the older person, and prevent unnecessary radiation exposure. A "COVID-19 blood battery", designed to increase the efficiency of evaluation of COVID-19 suspected patients is used in our institution. It includes D-dimers which are elevated in COVID-19 infections and potentially an index of severe infection. These 3 very frail patients presented late to the emergency department, all acutely and non-specifically unwell, with high prevalence of comorbidities and were transferred in by ambulance. They were triaged to the COVID-19 pathway of our hospital, and subsequently had negative COVID-19 swabs. All had an incidental finding of markedly elevated D-dimers, with potential causes of their symptoms other than pulmonary embolus. They were transferred to an acute geriatric ward specifically designated to manage older patients ( $>75 y e a r s)$ who had negative nasopharyngeal swab results. They were all ultimately diagnosed with extensive pulmonary emboli with evidence of raised pulmonary pressures on CTPA and/or echocardiogram. It is possible that these patients had false negative COVID-19 swabs. Allowing for the novel nature of COVID-19, prospective evaluation for new symptoms and complications such as thromboembolic disease in those affected by milder symptoms should be considered. In the absence of clinical improvement following treatment of other conditions in frail older patients, D-dimer testing could be indicated with pursuit of specific diagnostic evaluation for venous thromboembolism when significantly elevated.
\end{abstract}

Key words: Pulmonary embolus, COVID-19, D-dimer, older patient.

\section{Introduction}

D-dimer is routinely measured to exclude a diagnosis of venous thromboembolism (VTE) (1) and is the main biomarker of VTE (2). Appropriate age-adjusted D-dimer testing improves D-dimer specificity (3), and could decrease inappropriate CT pulmonary angiograms (CTPA) in the older person $(4,5)$, thus preventing unnecessary radiation exposure. Age-adjusted D-dimer is defined as; age $\mathrm{x} 10 \mu \mathrm{g} / \mathrm{l}$, in patients 50 years or over (6).

Recent European Society of Cardiology (ESC) guidelines (7) highlight the shortcomings of D-dimer testing with a low positive predictive value given that it rises in malignancy, inflammation, and hospitalised patients. The ESC endorse standardised clinical probability assessment, D-dimer testing and CTPA where appropriate.

A "COVID-19 blood battery"(CBB), is designed to efficiently evaluate and stratify suspected COVID-19 cases in our University teaching hospital (Table 1). It includes D-dimers which are elevated in COVID-19 infection and potentially an index of severe infection (8). Coagulopathy associated with COVID-19 infection is described (9) and thrombotic complications are common, especially in critically ill ICU patients with COVID-19 $(10,11)$.
Table 1

"COVID-19 blood battery" for routine workup of COVID-19

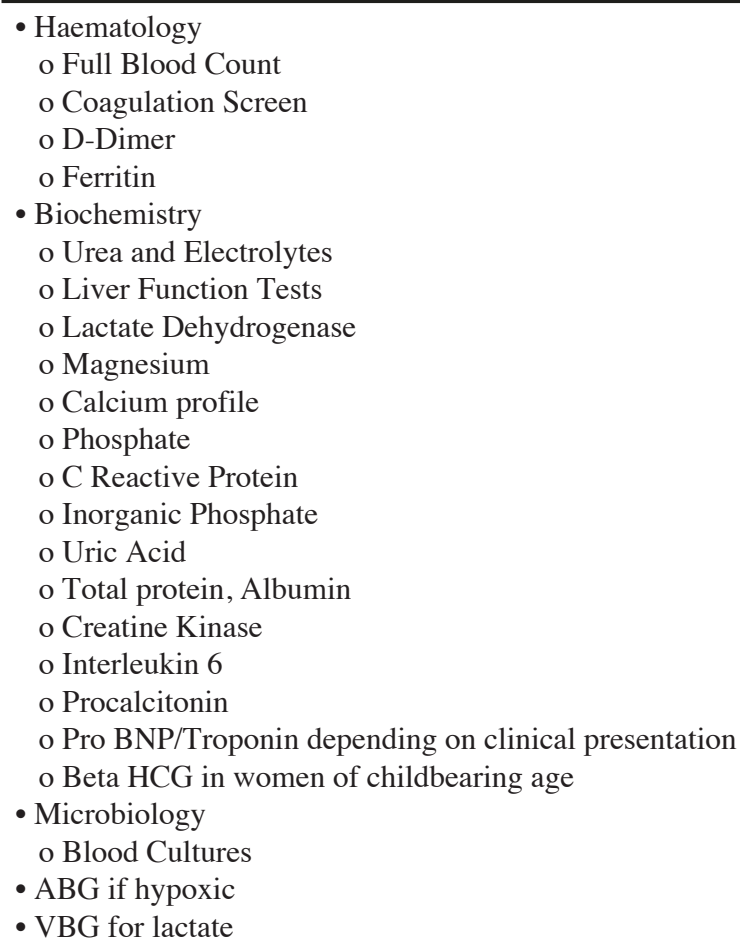




\section{THE JOURNAL OF NUTRITION, HEALTH \& AGING}

The authors describe 3 patients with initially suspected COVID-19 infection. All patients were admitted via the emergency department (ED), triaged as suspected COVID-19 cases, and transferred to a COVID-19 assessment ward. Wells score (WS) (12) was not evident in the medical notes. COVID19 was not detected, and they were subsequently transferred to an acute geriatric ward specifically designated to manage older patients ( $>75$ years) with negative nasopharyngeal swab results. All were diagnosed and treated for pathologies to account for their presentations to the ED, however in addition, all had unexpectedly elevated D-dimer levels on their CBB. This was followed by CTPA performed when there was no clinical improvement with preceding treatment. Pulmonary emboli (PE) with large clot burden and evidence of right heart strain were then confirmed. Written informed consent for publication of their clinical details was obtained from each patient.

\section{Case Histories}

\section{Case A}

An 89-year-old female nursing home resident was feeling generally unwell for 8 days and attended the ED following a temperature spike $\left(38^{\circ} \mathrm{C}\right)$ on 22 nd March. In the ambulance to $\mathrm{ED}$, she desaturated to $80 \%$. Heart rate at that time was 60 beats/minute, respiratory rate (RR) was $22 /$ minute and blood pressure (BP) 112/70 $\mathrm{mmHg}$. She denied any chest pain, dyspnoea, or palpitations. Her white cell count (WCC) was 22 x $10^{9} / \mathrm{L}$ with neutrophilia, and C Reactive Protein (CRP) was $126 \mathrm{mg} / \mathrm{L}$. Her lymphocyte count was normal and her chest $\mathrm{X}$-ray (CXR) was reported as demonstrating no focal infiltrates. Prothrombin Time (PT) was prolonged at 13 seconds and Activated Partial Thromboplastin Time (APTT) was reduced at 21.8 seconds and fibrinogen level was raised at $5.4 \mathrm{~g} / \mathrm{L}$. She was treated with IV antibiotics but, despite resolution of pyrexia, reduction of WCC and CRP, she continued to complain of lassitude, weakness and tiredness. A D-dimer initially performed in her CBB was $2655 \mu \mathrm{g} / \mathrm{l}$. In view of this and lack of clinical progress, a CTPA was performed which showed significant bilateral pulmonary emboli with a right sided infarct and reversal of blood flow noted in the hepatic inferior vena cava (IVC) indicative of right heart strain. If calculated in ED, her WS was estimated as 0. Echocardiogram revealed raised Pulmonary Arterial Pressure (PAP) at $48 \mathrm{mmHg}$. Her Charlson Comorbidity Index(CCI) (13) score was 6 points and Clinical Frailty Scale (CFS) (14) score was 7.

\section{Case B}

An 88-year-old lady was unwell for 6 days and attended the ED on 14th April due to an episode of light-headedness. In the ambulance to ED, she desaturated to $82 \%$. The patient had a known background of pulmonary hypertension for which she was on long term home oxygen therapy at a rate of $1 \mathrm{~L} / \mathrm{min}$. On admission, she had an increased oxygen requirement of $3 \mathrm{~L} /$ min. Her WCC was $12.4 \times 10^{9} / \mathrm{L}$ with lymphocyte count reduced to $0.7 \times 10^{9} / \mathrm{L}$, neutrophilia and CRP was $66 \mathrm{mg} / \mathrm{L}$. Her proBNP level was $6580 \mathrm{ng} / \mathrm{L}$ and her coagulation screen showed PT prolonged to 13.1 seconds and normal APTT and fibrinogen levels. D-dimer initially performed in her CBB was $4049 \mu \mathrm{g} / 1$. Her CXR showed a $6 \mathrm{~cm}$ inflammatory infiltrate in the right lower zone and she was treated with IV antibiotics and IV diuretics. If calculated in ED, her WS was estimated at 0 . Due to persisting weakness and lassitude and the raised D-dimer, a clinical decision was made to perform a CTPA, which showed right main pulmonary artery embolism with gross dilatation of the right atrium and ventricle. Echocardiogram revealed raised PAP at $53 \mathrm{mmHg}$. Her CCI score was 4 points and CFS score was 6.

\section{Case C}

A 76-year-old lady was feeling unwell with sweating and weakness for 10 days and attended the ED on $21 \mathrm{st}$ April following an episode of pre-syncope consistent with symptoms of orthostatic hypotension. She had evidence of mild delirium against a background of excellent cognition. In the ambulance to ED, she desaturated to $84 \%$. Her CXR in the emergency department showed no clear consolidation. She had severe kyphoscoliosis. Her WCC was $14.2 \times 10^{9} / \mathrm{L}$ with reduced lymphocyte count of $0.9 \times 10^{9} / \mathrm{L}$, neutrophilia, and CRP was $24 \mathrm{mg} / \mathrm{L}$. Her coagulation screen was within the normal range. Troponin $\mathrm{T}$ was raised at $165 \mathrm{ng} / \mathrm{L}$ with a further increase to $277 \mathrm{ng} / \mathrm{L} 6$ hours later and she was treated for acute coronary syndrome. She was also treated with IV antibiotics and transferred to the ward. If calculated in ED, her WS was estimated at 1.5 points (heart rate $105 / \mathrm{min}$ ). D-dimer initially performed in her CBB was $>10,000 \mu \mathrm{g} / 1$. Because of the grossly elevated level and, crucially, based on the results of Cases A and B, a CTPA was performed which showed bilateral pulmonary emboli with reversal of blood flow noted in the hepatic IVC indicative of right heart strain. Echocardiogram revealed PAP estimated at $38 \mathrm{mmHg}$ (assuming that the right atrial pressure was $5 \mathrm{mmHg}$ ) but the right side of the heart was poorly visualised. Her CCI score was 3 points and CFS score was 7.

All 3 patients were treated with subcutaneous low molecular weight heparin followed by direct oral anticoagulant under the supervision of the Haematology team and made a good recovery with discharge from hospital following return to baseline.

\section{Discussion}

Older people are more vulnerable to poor outcomes from COVID-19 infection (15), and are currently appropriately assessed regarding the possibility of COVID-19 infection in the ED. These very frail patients were delayed in reporting of symptoms, were all acutely but non-specifically unwell, had a high prevalence of comorbidities and transferred by ambulance to the ED. They were triaged to the COVID-19 pathway of 


\section{OPPORTUNISTIC DIAGNOSIS OF EXTENSIVE PULMONARY EMBOLUS FOLLOWING “COVID-19 BLOOD BATTERY”}

our hospital, were assessed with a CBB and subsequently had negative COVID-19 swabs. All had an incidental finding of markedly elevated D-dimers and had potential causes of their symptoms other that PE. The WS was not documented in any of the medical case notes, supporting a lack of suspicion for $\mathrm{PE}$ on their initial presentation to the ED, and scores were low on retrospective estimation. They were all diagnosed with extensive PE on CTPA, (prompted by the markedly elevated D-dimer levels) with evidence of right-sided cardiac strain on CTPA and/or echocardiogram.

Markedly elevated D-dimer results are common amongst COVID-19 non-survivors and markers of severity of the inflammatory response (16). The hospital's innovative CBB including D-dimer testing is designed to efficiently evaluate and stratify suspected COVID-19 cases.

One of the reported patients (case B) had light-headedness and another had pre-syncope which was posture-related (case C). A PE can present with syncope, with varying estimates of its prevalence from $17 \%$ of selected hospitalised patients with first presentation of syncope in a prospective study to 0.15 $2.1 \%$ of hospitalised patients in a large retrospective study $(17,18)$. Neither of these patients had a history (or collateral history) of actual syncope nor had there been specific symptoms or signs of VTE. These non-specific symptoms would not represent an indication to pursue a diagnosis of PE in our opinion.

What are the implications of the diagnosis of an extensive $\mathrm{PE}$ causing right heart strain in these very frail older patients, mainly arising from a markedly elevated D-dimer level identified as part of an agreed battery of tests performed on all COVID-19 infection suspects in our institution? There are 2 possibilities. Firstly, given that false negative nasopharyngeal COVID-19 swabs can occur in up to $40 \%$ of cases (19) it is possible that these 3 patients had relatively mild COVID19 infections which is known to be associated with prothrombotic tendency in patients affected by severe infections resulting in ICU admission (9-11). All had prolonged mild and non-specific symptoms prior to admission, and thus could potentially have small viral loads with minimal contamination of nasopharyngeal areas, while having some indicators (e.g. low lymphocyte count, pulmonary infiltrates, cardiac symptoms) associated with COVID-19 infection. There is no data to suggest the prothrombotic tendency of severe COVID19 infection is also present in patients affected with relatively mild symptoms to our knowledge. A recently published case report of a nonagenarian with non-specific symptoms of low grade pyrexia, delirium (similar to Case $\mathrm{C}$ reported here) and abdominal pain diagnosed by post-mortem nasopharyngeal swab serves to emphasise the unpredictable nature of the COVID-19 syndrome as it affects our population (20). Thus our understanding will continue to unfold with increased experience especially in older frail patients. Allowing for the novel nature of COVID-19, prospective alertness to new symptoms and complications such as thromboembolic disease in those
COVID-19 patients affected by milder symptoms is merited.

Secondly, these may indeed be true negative swab results and in the absence of clinical improvement following treatment of other conditions in very frail older patients without COVID-19 infection, D-dimer testing may be appropriate and subsequent specific testing for VTE if elevated significantly. Further studies specifically in this frail population would be necessary to establish if such an approach is beneficial.

Conflicts of interest: None.

Ethical Standards: Informed consent from all patients.

\section{References}

1. Johnson ED, Schell JC, Rodgers GM. The D-dimer assay. Am J Hematol 2019;94(7):833-9.

2. Riva N, Vella K, Hickey K, Bertù L, Zammit D, Spiteri S, et al. Biomarkers for the diagnosis of venous thromboembolism: D-dimer, thrombin generation, procoagulant phospholipid and soluble P-selectin. J Clin Pathol. 2018;71(11):1015-22.

3. Farm M, Siddiqui A, Onelöv L, Järnberg I, Eintrei J, Maskovic F, et al. Age-adjusted D-dimer cut-off leads to more efficient diagnosis of venous thromboembolism in the emergency department: a comparison of four assays. J Thromb Haemost. 2018;16(5):866-75.

4. Lozano-Polo L, Puig-Campmany M, Herrera-Mateo S, Mateo-Roca M, SantosRodríguez J, Benito-Vales S. Diagnosis of pulmonary embolism in the elderly: adherence to guidelines and age-adjusted D-dimer concentration values. Emergencias. 2018;30(5):321-7.

5. Thachil J, Lippi G, Favaloro EJ. D-dimer testing: laboratory aspects and current issues. Methods Mol Biol. 2017;1646:91-104.

6. Righini M, Van Es J, Den Exter PL, Roy P-M, Verschuren F, Ghuysen A, et al. Ageadjusted D-dimer cutoff levels to rule out pulmonary embolism: the ADJUST-PE study. JAMA. 2014;311(11):1117-24.

7. Konstantinides SV, Meyer G, Becattini C, Bueno H, Geersing G-J, Harjola V-P, et al. ESC Guidelines for the diagnosis and management of acute pulmonary embolism developed in collaboration with the European Respiratory Society (ERS) The Task Force for the diagnosis and management of acute pulmonary embolism of the European Society of Cardiology (ESC). Eur Heart J. 2020;41(4):543-603.

8. Kollias A1 KK, Dimakakos E1, Poulakou G1, Stergiou GS1, Syrigos K1 Thromboembolic risk and anticoagulant therapy in COVID-19 patients: Emerging evidence and call for action. Br J Haematol. 2020 Apr 18. doi: 10.1111/bjh.16727. [Epub ahead of print].

9. Thachil J, Tang N, Gando S, Falanga A, Cattaneo M, Levi M, et al. ISTH interim guidance on recognition and management of coagulopathy in COVID-19. J. Thromb. Haemost. 2020 Mar 25; [Epub ahead of print].

10. Klok F, Kruip M, Van der Meer N, Arbous M, Gommers D, Kant K, et al. Incidence of thrombotic complications in critically ill ICU patients with COVID-19. Thromb Res. 2020 Apr 10. pii: S0049-3848(20)30120-1. doi: 10.1016/j.thromres.2020.04.013. [Epub ahead of print].

11. Cui S, Chen S, Li X, Liu S, Wang F. Prevalence of venous thromboembolism in patients with severe novel coronavirus pneumonia. J Thromb Haemost. 2020 Apr 9. doi: $10.1111 /$ jth.14830. [Epub ahead of print].

12. Wells PS, Anderson DR, Rodger M, Ginsberg JS, Kearon C, Gent M, et al. Derivation of a simple clinical model to categorize patients probability of pulmonary embolism: increasing the models utility with the SimpliRED D-dimer. Thromb Haemost. 2000;83(03):416-20.

13. Charlson ME, Pompei P, Ales KL, MacKenzie CR. A new method of classifying prognostic comorbidity in longitudinal studies: development and validation. J Chronic Dis. 1987;40(5):373-83.

14. Rockwood K, Song X, MacKnight C, Bergman H, Hogan DB, McDowell I, et al. A global clinical measure of fitness and frailty in elderly people. CMAJ. 2005;173(5):489-95.

15. Porcheddu R, Serra C, Kelvin D, Kelvin N, Rubino S. Similarity in case fatality rates (CFR) of COVID-19/SARS-COV-2 in Italy and China. J Infect Dev Ctries. 2020;14(02):125-8

16. Tang N, Li D, Wang X, Sun Z. Abnormal coagulation parameters are associated with poor prognosis in patients with novel coronavirus pneumonia. J Thromb Haemost. 2020 Apr;18(4):844-847. doi: 10.1111/jth.14768. Epub 2020 Mar 13.

17. Prandoni P, Lensing AW, Prins MH, Ciammaichella M, Perlati M, Mumoli N, et al 


\section{THE JOURNAL OF NUTRITION, HEALTH \& AGING}

Prevalence of pulmonary embolism among patients hospitalized for syncope. N Engl J Med. 2016;375:1524-31.

18. Costantino G, Ruwald MH, Quinn J, Camargo CA, Dalgaard F, Gislason G, et al Prevalence of pulmonary embolism in patients with syncope. JAMA Intern Med. 2018;178(3):356-62.

19. Wang W, Xu Y, Gao R, Lu R, Han K, Wu G, et al. Detection of SARS-CoV-2 in different types of clinical specimens. JAMA. 2020 Mar 11. doi: 10.1001/ jama.2020.3786. [Epub ahead of print].
20. Tay HS, Harwood R. Atypical presentation of COVID-19 in a frail older person. Age Ageing. 2020 Apr 21. pii: afaa068. doi: 10.1093/ageing/afaa068. [Epub ahead of print]. 